\title{
Carbon dioxide: Signal for Excystment of Naegleria gruberi
}

\author{
By M. AVERNER* AND C. FULTON \\ Department of Biology, Brandeis University, Waltham, \\ Massachusetts 02154, U.S.A.
}

(Received 10 June 1965)

\begin{abstract}
SUMMARY
Exposure of cysts of Naegleria gruberi to slightly increased environmental $\mathrm{CO}_{2}$ caused excystment. Excystment was also induced by addition of proline or by an increase in the cyst population density, but both did so by increasing the amount of $\mathrm{CO}_{2}$ produced by the bacteria (Aerobacter aerogenes) which contaminated the cyst suspensions. Molecular $\mathrm{CO}_{2}$ would seem to be an excellent signal to induce excystment of a phagotrophic soil amoeba since the presence of $\mathrm{CO}_{2}$ would indicate an environment favourable for growth of the amoebae. Once excystment is initiated, it can proceed to completion in atmospheric $\mathrm{CO}_{2}$.
\end{abstract}

\section{INTRODUCTION}

An adaptive environmental signal for excystment should lead to emergence of the organism into an environment in which there would be a high probability of survival and growth. In the laboratory numerous stimuli can induce the excystment of protozoan cysts. These stimuli include shifts in temperature (Johnson \& Evans, 1940) and $\mathrm{pH}$ value (Darby, 1929), the addition of water (Garnjobst, 1928) or of chemicals such as carbohydrates (Barker \& Taylor, 1933), glycine (Beers, 1945), dicarboxylic acids and acetate (Haagen-Smit \& Thimann, 1938), or viable bacteria (Crump, 1950). This diversity of stimuli might reflect the variety of organisms studied, each with its specific stimulus, a variety of stimuli for a given organism or laboratory artifacts. Certain observations are particularly suggestive. Colpoda excysts in response to all the chemicals listed above, to bacteria (Singh, 1941), and even to appropriate agitation (Barker \& Taylor, 1933). One frequent stimulus, the presence of viable bacteria, causes numerous changes in the environment, only one of which may be the signal for excystment. In addition, viable bacteria, often of unknown type and unknown quantity, are usually present with the other agents studied, and possibly alter the added agent in an unknown way. The effect of bacteria on chemically induced excystment has usually been ignored or disposed of with a statement such as 'in all crucial experiments the cysts become active long before the bacteria could possibly produce an effect' (Beers, 1945).

Naegleria gruberi is a small phagotrophic soil amoeba which under appropriate conditions forms cysts or transforms into flagellates. Though the stimulus for naegleria excystment has not been studied, excystment has been described (Wilson, 1916; Rafalko, 1947; Schuster, 1963). One of the most dramatic cytological

* Present address: Department of Molecular Biology and Biophysics, Yale University, New Haven, Connecticut, U.S.A. 
changes during excystment is the reappearance of contractile vacuoles, which are not present in the uninduced cyst. Active amoeboid motion precedes the emergence of the amoeba through one of several pores in the cyst wall. For our purposes a cyst is considered to be 'full' as long as the cytoplasm is entirely within the cyst wall, but once emergence begins and the cytoplasm is partially within and partially without, the cyst is 'transitional'. The emerging amoeba leaves behind an empty cyst wall. The excysted amoebae develop flagella, but this is without apparent effect on excystment.

This paper describes a study of three environmental variables-proline, $\mathrm{CO}_{2}$, cyst population density-which induce the excystment of Naegleria gruberi, and of the role of bacteria in the process.

\section{METHODS}

Growth and preparation of cysts. Naegleria gruberi NB-1 and the details of procedures used for cultivating the amoebae and performing counts will be described by Fulton \& Dingle (to be published). Amoebae were grown in association with Aerobacter aerogenes on autoclaved agar medium, NM, containing (g./l. distilled water): Difco Bacto-peptone, $2 \cdot 0$; glucose, $2 \cdot 0 ; \mathrm{K}_{2} \mathrm{HPO}_{4}, 1 \cdot 5 ; \mathrm{KH}_{2} \mathrm{PO}_{4}, 1 \cdot 0 ;$ Difco Bacto-agar, 20. About $10^{5}$ cysts and $0 \cdot 1 \mathrm{ml}$. of an overnight broth culture of Aerobacter aerogenes were spread over the surface of NM agar in a $100 \mathrm{~mm}$. diam. Petri plate, and the plate incubated at 33-34. Under these conditions the cysts excysted, and the resulting amoebae grew exponentially until they reached stationary phase at 2-3 $\times 10^{7}$ amoebae/plate. Some amoebae began to encyst after about $20 \mathrm{hr}$ and all encysted within $48 \mathrm{hr}$. The plates of cysts were stored at $34^{\circ}$ and used 4-6 days after plating.

Cysts were prepared for excystment experiments by harvesting each plate into $10 \mathrm{ml}$. demineralized water and centrifuging the suspension at $10^{\circ}$ for 105 sec. at $500 \mathrm{~g}$ in a swinging bucket rotor. The supernatant fluid was discarded and the pellet washed twice more in demineralized water. The final pellet was resuspended under conditions suitable to the experiment. The cyst concentration was determined by a Coulter counting apparatus (Coulter Electronics, Hialeah, Florida, U.S.A.) and the bacteria by colony count.

Excystment on agar. Agar excystment experiments were done on autoclaved agar media containing $2 \%(\mathrm{w} / \mathrm{v})$ Bacto agar and supplements as indicated for each experiment. A sample of a washed counted cyst suspension was spread over the surface of a plate, and the plate placed, agar upward, without its cover, in a desiccator of about $2 \mathrm{l}$. capacity which contained $50 \mathrm{ml}$. of a $10 \%(\mathrm{w} / \mathrm{v}) \mathrm{NaCl}$ solution to maintain constant humidity. The gas phases were adjusted (see below), and the desiccators incubated at $34^{\circ}$. The beginning of incubation was the zero time for each experiment.

To evaluate excystment on agar, the organisms were examined in situ. The plates were removed from the desiccators and a circular coverslip $18 \mathrm{~mm}$. in diameter was placed directly on the agar, with care taken to allow a film of liquid to flow between the agar and the coverslip. The organisms were examined with phase contrast optics at a magnification of $\times \mathbf{4 0 0}$. When the plates were to be re-incubated they were returned to the desiccators and the gas phases readjusted. For each time point a fresh area of a plate was examined. 
Excystment in liquid. Liquid excystment experiments were normally done in demineralized water containing either $2 \times 10^{-3} \mathrm{M}$ tris buffer $(\mathrm{pH} 7 \cdot 4)$ or $10^{-3} \mathrm{M}$ potassium phosphate buffer ( $\mathrm{pH} 7 \cdot \mathbf{7})$. Unless otherwise stated, a sample of a washed and counted cyst suspension was diluted into $10 \mathrm{ml}$. of buffer in a $125 \mathrm{ml}$. Erlenmeyer flask. The flask was sealed with a serum vial stopper, its gas phase adjusted, and then shaken at $30^{\circ}$ in a water bath at the rate of seventy 1 -inch strokes $/ \mathrm{min}$. The beginning of incubation was taken as the zero time of the experiment.

Evaluation of excystment in liquid. At intervals $\mathbf{0 . 2} \mathrm{ml}$. samples of the cyst suspension were removed from the flasks by using a $1 \mathrm{ml}$. syringe with a 5 -inch 20-gauge needle. The cysts in the samples were fixed and stained by injecting the samples into tubes containing one drop of a 1/10 dilution of stock Lugol's iodine (Fulton \& Dingle, to be published) in distilled water. The fixed samples were examined with phase contrast optics at $\times \mathbf{4 0 0}$, and classified as full, transitional and empty cysts (amoebae being ignored). The process of excystment can be measured as either percent full or percent transitional and empty cysts; full cysts were used. Each determination represented a count of a sample of 100 cysts. Measurements of reconstructed populations of cysts and amoebae indicated that clumping or other events capable of yielding inaccurate counts did not occur during incubation, sampling and fixing, or slide preparation.

Adjustment of the $\mathrm{pCO}_{2}$. Desiccators or flasks were evacuated by aspiration, a measured volume of $\mathrm{CO}_{2}$ gas (Matheson 'Bone Dry' grade, minimum purity 99.8 \% $\mathrm{CO}_{2}$ ) injected from a syringe, and water-washed air allowed to enter the vessel until atmospheric pressure was reached (see Loomis, 1959). The $\mathrm{pCO}_{2}$ was calculated as the percent of the total volume of the vessel occupied by the volume of $\mathrm{CO}_{2}$ injected. The $\mathrm{pCO}_{2}$ of the laboratory atmosphere was assumed to be $0.03 \%$.

To compare excystment in the presence and absence of $\mathrm{CO}_{2}$, cysts were incubated in absorption flasks fitted with centre wells containing paper wicks and either $1 \mathrm{ml}$. of distilled water or $1 \mathrm{ml}$. of $20 \%(\mathrm{w} / \mathrm{v}) \mathrm{KOH}$. Warburg vessels shaken at 140 strokes per minute were used in some experiments to improve gas exchange. To guard against spillage of $\mathrm{KOH}$, the $\mathrm{pH}$ of the excystment solution was measured at the end of each experiment.

\section{RESULTS}

\section{Inducers of naegleria excystment}

Proline. In the course of other work, proline was observed to induce the excystment of Naegleria gruberi. Liquid suspensions of cysts excysted when L-proline was added at concentrations greater than $10^{-5} \mathrm{M}$; the rate of excystment was similar and maximal at $10^{-4}, 10^{-3}$, and $10^{-2} \mathrm{M}$. The excysted amoebae immediately transformed into flagellates, and to avoid this we attempted to obtain excystment on agar plates where transformation does not occur. Contrary to the effect of proline in liquid, cysts placed on proline agar did not excyst, regardless of the concentration of proline. Extending the time of incubation, washing the agar, or varying the number of cysts plated did not result in excystment. The disparity between the results in liquid and on agar was resolved by a chance observation. On proline agar plates, uncovered cysts remained dormant while those under a coverslip excysted after an hour (Fig. 1). It seemed likely that the coverslip 
created an environment stimulating excystment by interfering with gas exchange. One possibility was an increase in $\mathrm{CO}_{2}$ concentration under the coverslip.

Carbon dioxide. Proline agar plates spread with cysts were incubated in atmospheres of different $\mathrm{CO}_{2}$ concentrations (Table 1 ). Increased $\mathrm{pCO}_{2}$ induced excystment, and the amount of excystment was a function of the $\mathrm{pCO}_{2}$. Furthermore, when the $\mathrm{pCO}_{2}$ was increased, excystment occurred independently of the presence of proline in the agar.

In liquid, where measurement of degree of excystment is much more precise, either proline or $\mathrm{CO}_{2}$ induced excystment (Fig. 2). Under similar conditions of temperature and aqueous environment and with saturating concentrations of

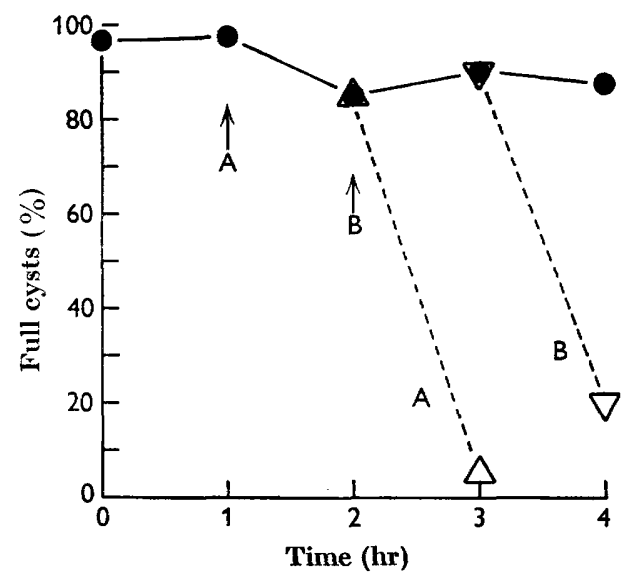

Fig. 1

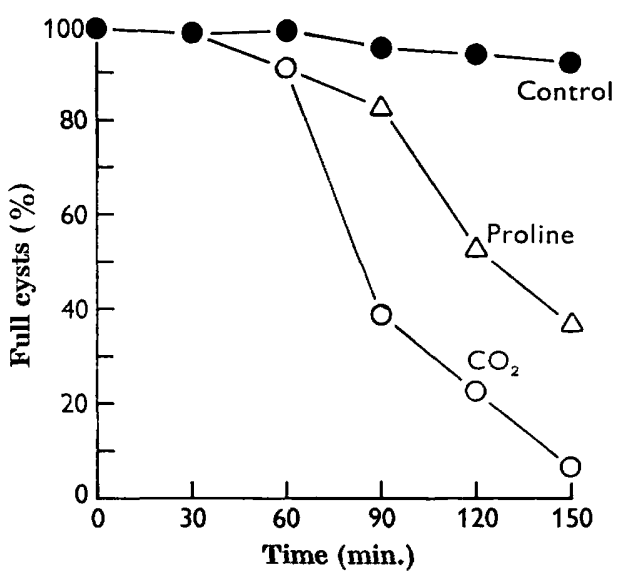

Fig, 2

Fig. 1. The coverslip experiment. An agar plate buffered at $\mathrm{pH} \mathrm{7.4}$ with $0.02 \mathrm{M}$-tris and containing $10^{-3} \mathrm{M}-\mathrm{L}$-proline was spread with $2 \times 10^{6}$ cysts and incubated at $34^{\circ}$ under standard conditions. A sample of the initial cyst suspension was fixed and counted. At hourly intervals a coverslip was applied to a new area of the plate and excystment evaluated in situ; at the same time the areas under the coverslips applied at one (A) and at $2 \mathrm{hr}$ (B) were re-examined.

Fig. 2. Induction of excystment in liquid by proline and by $\mathrm{CO}_{2}$. Three flasks were prepared each containing $5 \mathrm{ml}$. of phosphate buffer, pH 6.8, with $8 \times 10^{5}$ cysts per ml. One flask also contained $10^{-3} \mathrm{M}$-proline, and another flask was capped and its atmosphere adjusted to $0.95 \% \mathrm{pCO}_{2}$. The flasks were shaken at $25^{\circ}$ with 100 strokes per minute. At the end of the experiment the $\mathrm{pH}$ in the flasks ranged from 6.85 for the control to 6.7 for the flask with increased $\mathrm{pCO}_{2}$.

\section{Table 1. Excystment on proline agar in varying $\mathrm{CO}_{2}$ atmospheres}

Washed cysts were spread at $2 \times 10^{6}$ cysts per plate on agar plates buffered at $\mathrm{pH} 6 \cdot 8$ with 0.02 M-potassium phosphate and containing $10^{-3}$ M-L-proline. The plates were placed in desiccators, the atmospheres adjusted to various $\mathrm{CO}_{2}$ concentrations, and the desiccators incubated at $34^{\circ}$. Excystment was evaluated after $4 \mathrm{hr}$ incubation.

\begin{tabular}{lccrr} 
& \multicolumn{4}{c}{$\mathrm{pCO}_{2}(\%)$} \\
Full cysts & 0.03 & 0.33 & $1 \cdot 0$ & $2 \cdot 0$ \\
Transitional cysts & 90 & 50 & 5 & 0 \\
Empty cysts & 3 & 15 & 5 & 7 \\
& 7 & 35 & 90 & 93
\end{tabular}


$\mathrm{CO}_{2}$ or proline, excystment regularly occurred sooner in response to $\mathrm{CO}_{2}$ than to proline.

Measurement of the response to $\mathrm{CO}_{2}$ depended on having a measure of the relative rate of excystment. If excystment of a population were synchronous, there would be an abrupt transition from full cysts to transitional cysts and then to empty cysts. Excystment under the conditions we used was asynchronous, and a plot of the $\%$ full cysts against time of incubation gave a sigmoid curve. If such a curve represented a summed normal distribution of excystment times, the probit transformation would convert it to a linear curve (Finney, 1962). Probability curves for naegleria excystment approximate linearity (Fig. 3). The mean time of

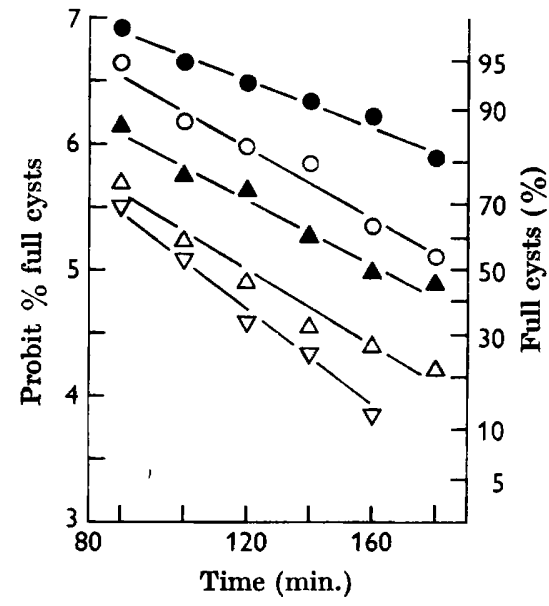

Fig. 3

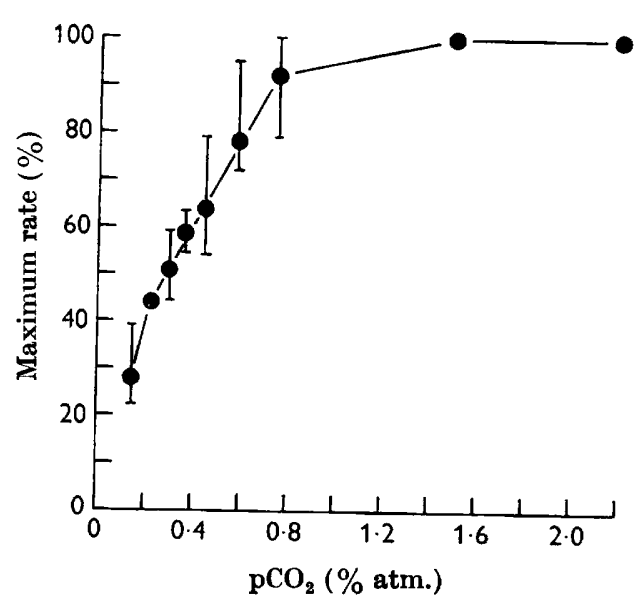

Fig. 4

Fig. 3. Excystment at various $\mathrm{CO}_{2}$ concentrations. Washed cysts were suspended at a concentration of $1 \times 10^{5}$ per ml. in $10 \mathrm{ml}$. of phosphate buffer in $125 \mathrm{ml}$. flasks, the atmosphere of the flasks adjusted, and the flasks shaken at $25^{\circ}$ at 70 strokes per minute. The $\mathrm{CO}_{2}$ concentrations of the atmospheres were: $0,0.15 ; 0,0.30 ; \Delta, 0.44$; $\triangle, 0.59 ; \nabla, 0.74 \%$.

Fig. 4. Excystment as a function of $\mathrm{CO}_{2}$ concentration. Conditions for excystment were described in Fig. 3, and the relative rate of excystment was the reciprocal of the time required for $50 \%$ excystment (obtained from probability curves). Where several curves were obtained at the same $\mathrm{pCO}_{2}$, the mean value with the range is plotted.

excystment (the time for $50 \%$ excystment) varied with the $\mathrm{pCO}_{2}$ (Fig. 3). The reciprocal of the mean excystment time was used as a measure of the relative rate of excystment (this approach, used with a similar morphological change, will be discussed in Fulton \& Dingle, to be published). Though the mean excystment time varied with $\mathrm{pCO}_{2}$, the slopes of the probability curves, which represent the standard deviation did not vary greatly, so the heterogeneity of the response of the cyst populations, to increased $\mathrm{pCO}_{2}$ was not markedly affected by the relative rate of excystment.

The effect of $\mathrm{CO}_{2}$ concentration on the relative rate of excystment was determined by multiple measurements under standardized conditions (Fig. 4). The response of cysts was a function of $\mathrm{pCO}_{2}$ in the range from $0 \cdot 15 \%$ to about $1 \%$. Excystment at any $\mathrm{pCO}_{2}$ less than $0 \cdot 15 \%$ was too slow and variable to obtain valid 
measurements of rate. Thus we do not know whether a threshold level of $\mathrm{pCO}_{2}$ (i.e. above atmospheric) is necessary to induce excystment. Cysts can be stored on agar plates in atmospheric $\mathrm{pCO}_{2}$ for prolonged periods without significant excystment; this suggests that excystment does not occur unless $\mathrm{pCO}_{2}$ exceeds $0.03 \%$.

A portion of the $\mathrm{CO}_{2}$ dissolving in an aqueous solution ionizes to increase the $\mathrm{H}^{+}, \mathrm{HCO}_{3}{ }^{-}$, and $\mathrm{CO}_{3}=$ content of the solution. Is molecular $\mathrm{CO}_{2}$ the stimulus for excystment, or is one of its ionic products the effective agent? Acid pH values have been reported to induce excystment (Darby, 1929), but naegleria cysts placed in phosphate buffers from $\mathrm{pH} \mathrm{3.3}$ to 7 did not excyst until $\mathrm{CO}_{2}$ was added.

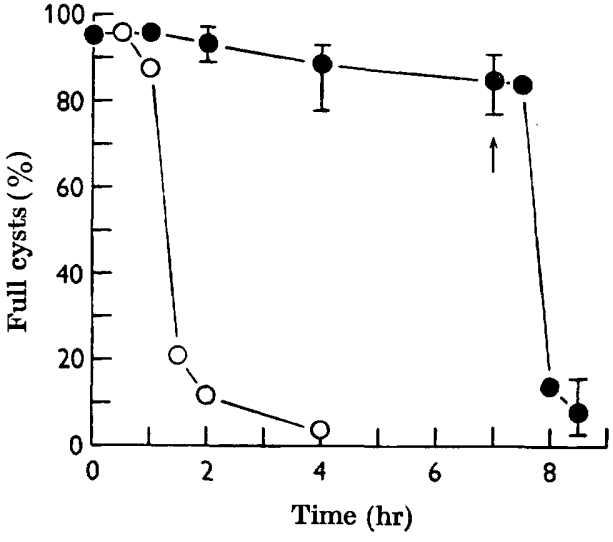

Fig. 5

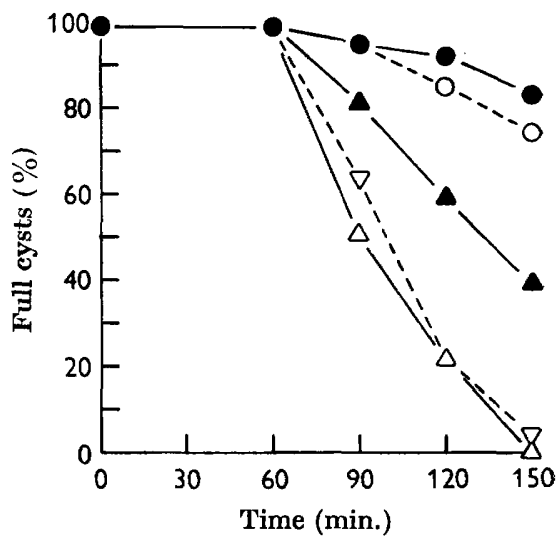

Fig. 6

Fig. 5. Effect of bicarbonate on excystment. Washed cysts were suspended at $10^{5}$ per $\mathrm{ml}$. in phosphate buffer containing various concentrations of bicarbonate and shaken at $30^{\circ}$ with 70 strokes per minute. The closed circles give the mean values with the range, for four flasks containing $10^{-2}, 10^{-3}, 10^{-4}$, and $10^{-5} \mathrm{M}$-bicarbonate. A flask was included without bicarbonate but containing $0.8 \% \mathrm{pCO}_{2}$ (open circles). At the point indicated by the arrow the bicarbonate flasks were capped and $0.8 \% \mathrm{pCO}_{2}$ added to each.

Fig. 6. Density-induced excystment. Washed cysts were suspended in tris $(\mathrm{pH} 7 \cdot 4)$, and $10 \mathrm{ml}$. samples shaken at $25^{\circ}$ in flasks capped with serum vial stoppers. The numbers of cysts $\times 10^{5}$ per ml. were: $\bullet, 1 \cdot 1 ; 0,3 \cdot 3 ; \Delta, 11 ; \Delta, 23 ; \nabla, 55$.

In the buffered solutions used the addition of $\mathrm{CO}_{2}$ did not markedly alter the pH value (e.g. Fig. 2), so changes in $\mathrm{pH}$ value are not responsible for excystment. Bicarbonate ions also did not induce excystment (Fig. 5); at high concentrations the slight excystment observed was probably due to molecular $\mathrm{CO}_{2}$ produced in the solution. The addition of $\mathrm{CO}_{2}$ to the bicarbonate solutions induced excystment (Fig. 5), showing that bicarbonate neither stimulated nor inhibited the process. Carbonate ions, as well as carbonic acid, are produced in very small amounts at the neutral $\mathrm{pH}$ value used for excystment. Carbonate should be produced in similar or greater amounts in the bicarbonate solutions, which did not induce excystment. Also, changing the $\mathrm{pH}$ value of the excystment environment, and thus markedly altering the proportions of carbonic acid and carbonate (see Scott, 1939), did not markedly affect the rate of excystment in response to $\mathrm{CO}_{2}$. For example, the relative rate of excystment in response to $0.6 \% \mathrm{pCO}_{2}$ in phosphate buffered solutions 
at $\mathrm{pH} 5$ and $\mathrm{pH} 9$ was about $70 \%$ of the rate at $\mathrm{pH} 7$. Thus by elimination we conclude that dissolved molecular $\mathrm{CO}_{2}$ gives the signal for excystment.

Effect of cyst population density. In a study of the effect of cyst population density on the relative rate of excystment in response to $\mathrm{CO}_{2}$, we found that increasing the population density in liquid eventually led to excystment at atmospheric $\mathrm{pCO}_{2}$. The relative rate of population density-induced excystment was a function of the population density (Fig. 6). Considerable variability was encountered in the value of the population density required to obtain excystment with different batches of cysts.

\section{Comparison of inducers of excystment}

The addition of proline and an increase in the cyst population density both appeared to induce excystment by increasing the amount of $\mathrm{CO}_{2}$ in the cyst environment. In both cases the Aerobacter aerogenes present in washed cyst suspensions are believed responsible for the $\mathrm{CO}_{2}$ production. Proline can be decarboxylated or metabolized to $\mathrm{CO}_{2}$ by $A$. aerogenes. If uninduced cysts are metabolically inactive, the aerobacter would be responsible for the metabolism of proline to $\mathrm{CO}_{2}$. In liquid media enough $\mathrm{CO}_{2}$ to induce excystment is produced from the stimulation of aerobacter metabolism by proline, but on agar plates the $\mathrm{CO}_{2}$ produced diffuses away and excystment occurs only when gas exchange is prevented. Increasing the population density of cysts increases the number of aerobacters $/ \mathrm{ml}$., and thereby the amount of $\mathrm{CO}_{2}$ produced $/ \mathrm{ml}$. by the bacteria. If these assumptions are correct, $\mathrm{CO}_{2}$ is the active agent in all cases. Elimination of the bacteria, or removal of the $\mathrm{CO}_{2}$ produced by them, should prevent excystment in response to proline or density.

The $\mathrm{pCO}_{2}$ of the cyst environment can be decreased by absorbing $\mathrm{CO}_{2}$ from the gas phase of the flask with $\mathrm{KOH}$. $\mathrm{CO}_{2}$-induced excystment did not occur in the presence of alkali absorbant. Similarly, excystment in response to increased cyst population density or added proline was inhibited by removal of $\mathrm{CO}_{2}$ from the gas phase. KOH inhibition of excystment was dependent on sufficient shaking to ensure rapid gas exchange between liquid and gas phases. No excystment occurred in response to any inducers when $\mathrm{KOH}$ was present, whereas simultaneous controls without $\mathrm{KOH}$ did excyst.

A small amount of $\mathrm{CO}_{2}$ (e.g. atmospheric concentration) might be essential for excystment in response to the environmental alteration produced by proline or density. This possibility, as well as the role of the aerobacter, can be tested by allowing the $\mathrm{pCO}_{2}$ to remain at atmospheric levels but preventing the production of additional $\mathrm{CO}_{2}$ by the bacteria. Ideally this could be achieved by studying the excystment of sterile cysts (free of metabolizing as well as colony forming bacteria), but many attempts to sterilize cysts or to obtain viable cysts by sterile cultivation of amoebae were unsuccessful. (Cysts were formed in sterile cultures on autoclaved aerobacter, but they had abnormal morphology and excysted in liquid without any stimulus.) Because of these difficulties, partial sterilization was accomplished with streptomycin, to which Aerobacter aerogenes is sensitive. Overnight incubation of washed cysts on NM containing $200 \mu \mathrm{g}$. streptomycin $/ \mathrm{ml}$. lowered the population of viable aerobacter from about $10^{7}$ to less than $10^{3}$ per $10^{7}$ cysts plated. These cysts did not appear to be affected by streptomycin, and excysted 
normally in response to $\mathrm{CO}_{2}$. They did not, however, excyst at population densities which normally induce the excystment of untreated cysts. Streptomycin treatment also inhibited proline-induced excystment, though when streptomycin and proline were added to washed cysts simultaneously, slow excystment still occurred, suggesting that the bacteria, while unable to form colonies, were still able to metabolize proline to $\mathrm{CO}_{2}$.

The inhibition by $\mathrm{KOH}$ of $\mathrm{CO}_{2-}$, proline-, and density-induced excystment produces strong support for the hypothesis that increased $\mathrm{pCO}_{2}$ is the stimulus for excystment in all cases. The inhibition of proline- and density- but not $\mathrm{CO}_{2}$-induced excystment by streptomycin treatment of cysts indicates that the bacteria which contaminate the cyst suspensions are responsible for the $\mathrm{CO}_{2}$ production.

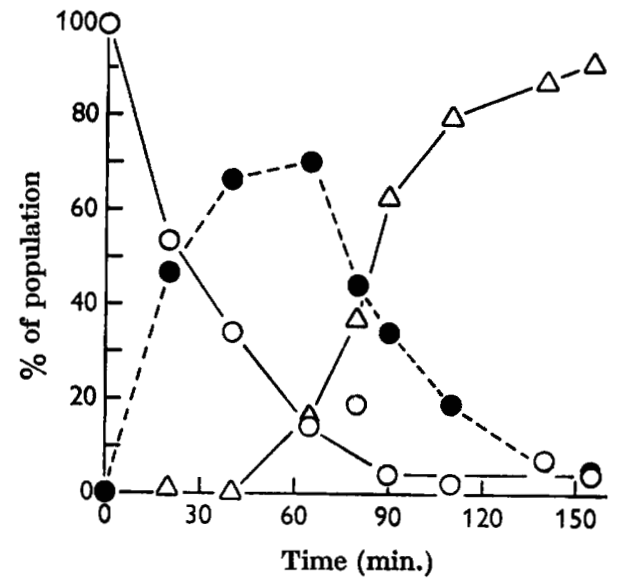

Fig. 7

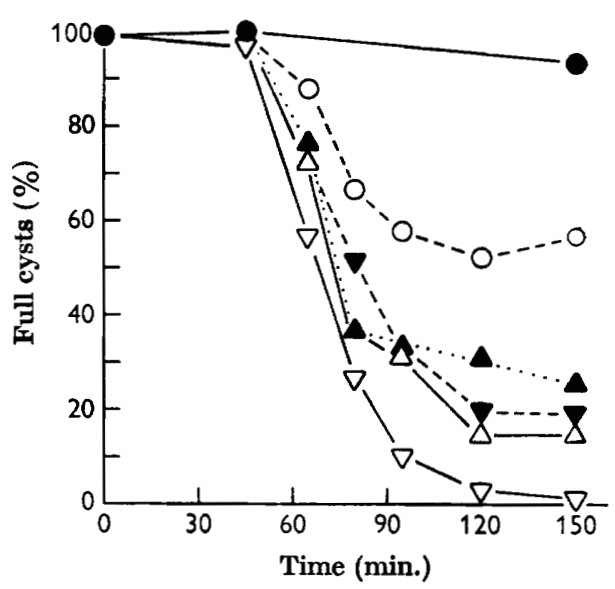

Fig. 8

Fig. 7. Temporal sequence of morphological changes during excystment. Ten ml. of washed cysts at $5 \times 10^{5}$ per $\mathrm{ml}$. in phosphate buffer $(\mathrm{pH} 7 \cdot 7)$ were shaken at $30^{\circ}$ in $2.4 \% \mathrm{pCO}_{2}$. At intervals samples of 100 cells were classified under phase contrast into the arbitrary stages described in the text: 0 , stage $1 ; 0$, stage $2 ; \Delta$, transitional and empty cysts.

Fig. 8. Influence of duration of exposure to $\mathrm{CO}_{2}$ upon subsequent excystment. Washed cysts at $3 \times 10^{5}$ per $\mathrm{ml}$. in phosphate buffer were distributed in $10 \mathrm{ml}$. samples to six flasks. One flask was left open (e), and the remaining flasks adjusted to $0.8 \% \mathrm{pCO}_{2}$. The flasks were shaken at $30^{\circ}$, and at intervals a flask containing $\mathrm{CO}_{2}$ was removed, its contents centrifuged and resuspended in $10 \mathrm{ml}$. phosphate buffer, and shaking continued at atmospheric $\mathrm{pCO}_{2}$. In each case the transfer took about $3 \mathrm{~min}$. The cysts were in elevated $\mathrm{pCO}_{2}$ for: $O, 30 ; \triangle, 45 ; \triangle, 65 ; \nabla, 80$; and $\nabla, 150 \mathrm{~min}$.

\section{Response of cysts to $\mathrm{CO}_{2}$}

Phase contrast observation of living organisms revealed extensive cytoplasmic changes before excystment. In an uninduced or stage 1 cyst the cytoplasm was filled with small particles in active Brownian motion, and no contractile vacuoles were present. After exposure of cysts to $\mathrm{CO}_{2}$, large phase-dense granules appeared in the cytoplasm (stage 2). Under standard excystment conditions, half of the cysts changed from stages 1 to 2 within about $20 \mathrm{~min}$. (Fig. 7). Soon thereafter contractile vacuoles were formed, and amoeboid movement began. About an hour 
after a cyst changed from stages 1 to 2 , an amoeba emerged through one of the pores in the cyst, leaving behind an empty cyst wall (Fig. 7). Cysts can recognize $\mathrm{CO}_{2}$ in their environment within $20 \mathrm{~min}$. and begin the sequence of changes which lead to emergence.

In order to test whether the continual presence of increased $\mathrm{pCO}_{2}$ is required for excystment, or whether $\mathrm{CO}_{2}$ induced a reaction after which excystment can continue to completion independently of the presence of $\mathrm{CO}_{2}$, cysts were exposed to an inducing concentration of $\mathrm{CO}_{2}$ for varying times, and then shaken at atmospheric $\mathrm{CO}_{2}$. Thirty minutes of exposure to $\mathrm{CO}_{2}$ was sufficient to induce excystment of about $50 \%$ of a cyst population; the extent of excystment was a function of the time the cyst population was exposed to $\mathrm{CO}_{2}$ up to about an hour (Fig. 8). Exposure to $\mathrm{CO}_{2}$ therefore initiates a sequence of events which can continue to completion in the absence of excess $\mathrm{CO}_{2}$.

After about $30 \mathrm{~min}$. exposure to $\mathrm{CO}_{2}$, half the cysts had reached morphological stage 2 (Fig. 7) and could complete excystment independently of increased $\mathrm{pCO}_{2}$ (Fig. 8). This correlation suggests that the critical effect of $\mathrm{CO}_{2}$ is to cause some early event, associated with the appearance of large granules, which results in a commitment to excystment.

\section{DISCUSSION}

Our major conclusion is that the only stimulus in the environment that naegleria cysts recognize as a signal for excystment is molecular $\mathrm{CO}_{2}$. The fitness of this signal as a means for a phagotroph to recognize an environment in which food is available is obvious. The level of $\mathrm{pCO}_{2}$ in an environment such as soil is a function of the rate of production of $\mathrm{CO}_{2}-\mathrm{a}$ measure of the population density of organisms, the temperature, and other conditions influencing growth and metabolism-and the rate of loss of $\mathrm{CO}_{2}$ by diffusion. Because of this, $\mathrm{CO}_{2}$ would seem to be one of the few molecules whose concentration in soil would indicate when overall conditions would be favourable for naegleria amoebae to survive and grow. The water content of soil, its $\mathrm{pH}$ or temperature, or many of the stable organic molecules soil contains would be less sensitive signals of such an environment. This hypothesis, though it certainly does not consider the complexities of soil ecology, has a simplicity which makes it attractive. Furthermore, the levels of $\mathrm{CO}_{2}$ found in topsoils are sufficient to induce the excystment of naegleria (see Thimann, 1963).

Ballard (1958) found that $\mathrm{CO}_{2}$ induces germination of clover seeds. Loomis (see 1959, 1964), in a study of the role of increased $\mathrm{pCO}_{2}$ in controlling sexual differentiation of hydra, has drawn attention to the many biological processes that are influenced by $\mathrm{CO}_{2}$.

Other environmental variables induce the excystment of naegleria, but these appear to act by increasing the amount of $\mathrm{CO}_{2}$ produced by bacteria in the cyst environment. Proline- and density-induced excystment in the laboratory display the effect of environmentally produced $\mathrm{CO}_{2}$. Although cyst population density was the observed variable in density-induced excystment, it is the simultaneously altered bacterial population density that is responsible for excystment. With some cyst preparations, less responsive to density-induced excystment, excystment could be obtained by adding additional aerobacters. On the nutrient agar for naegleria, NM, it is the growth of aerobacter-and presumably the $\mathrm{CO}_{2}$ micro- 
environment created by this growth-which induces excystment of naegleria. In all cases, increased $\mathrm{pCO}_{2}$ accounts for excystment. From this point of view, as long as bacteria are present anything which increases their rate of $\mathrm{CO}_{2}$ productionproline, other chemicals, a temperature increase, etc.-would lead to excystment. In this connexion, it is of interest that the washed cyst suspensions used in our experiments are contaminated to the extent of about one viable aerobacter per cyst; one Aerobacter has roughly one thousandth the volume of a cyst. This relatively small quantity of aerobacter, when stimulated by proline or increased in population density, is able to produce enough $\mathrm{CO}_{2}$ to induce excystment.

Many published studies of excystment of other organisms could be explained in the same way-i.e. that the addition of bacteria, organic acids, etc., induces excystment via $\mathrm{CO}_{2}$. For example, Crump (1950), in a study of the ability of various bacteria to induce the excystment of a limax amoeba, stated that 'The unsuccessful attempts already recorded to induce excystment by placing cysts in culture fluid in which Aerobacter...had grown, but from which they were removed by centrifuging, suggests that the substances concerned may be so transient that they disappear as fast as they are formed, and only when there are living bacteria in the medium is enough of the stimulating material present to act successfully on the cysts'. In studying the induction of excystment of didinium by bacteria, Beers (1946) noted that 'the effective substances are highly unstable. They are probably produced at the surface of the bacteria by an enzyme system. They diffuse a short distance into the medium, where they induce excystment, but only if present in relatively high concentrations-i.e. the concentration of the bacteria must be high to induce excystment. Once in the medium the substances are rapidly altered or destroyed'. Such statements might apply to excystment induced by bacteria-produced $\mathrm{CO}_{2}$.

Most of this study is included in a dissertation submitted to the graduate faculty of Brandeis University by M.A. in partial fulfilment of the requirements for the degree of Doctor of Philosophy. This work was done during the tenure of a Jack Cohn predoctoral fellowship, and completed while M.A. held a postdoctoral traineeship from training grant T1HD22 of the Institute of Child Health and Infant Development of the National Institutes of Health. The research was supported by a grant from the National Science Foundation.

\section{REFERENCES}

Ballard, L. A. T. (1958). Studies of dormancy in the seeds of subterranean clover (Trifolium subterraneum L.). I. Breaking of dormancy by carbon dioxide and by activated carbon. Aust. J. Biol. Sci. 11, 246.

Barker, H. A. \& Taylor, C. V. (1933). Studies on the excystment of Colpoda cucullis. Physiol. Zool. 6, $12 \%$.

BeErs, C. D. (1945). Some factors affecting excystment in the ciliate Tillina magna. Physiol. Zool. 18, 80.

BeErs, C. D. (1946). Excystment in Didinium nasutum, with special reference to the role of bacteria. J. exp. Zool. 103, 201.

Crump, L. M. (1950). The influence of the bacterial environment on the excystment of amoebae from soil. J. gen. Microbiol. 4, 16.

DARBY, H. H. (1929). The effects of the hydrogen ion concentration on the sequence of protozoan forms. Arch. Protistenk. 65, 1. 


$$
\mathrm{CO}_{2} \text {-induced excystment of naegleria }
$$

Finney, D. J. (1962). Probit Analysis: A Statistical Treatment of the Sigmoid Response Curve, 2nd ed. Cambridge: University Press.

GarnJobst, L. (1928). Induced encystment and excystment in Euplotes taylori, sp.nov. Physiol. Zool. 1, 561.

Hangen-Smit, A. J. \& Thrmann, K. V. (1938). The excystment of Colpoda cucullus. I. The chemical nature of the excysting factors in hay infusion. J. cell. comp. Physiol. $11,389$.

Johnson, W. H. \& Evans, F. R. (1940). Environmental factors affecting cystment in Woodruffia metabolica. Physiol. Zool. 13, 102.

Loomis, W. F. (1959). Feedback control of growth and differentiation by carbon dioxide tension and related metabolic variables. In Cell, Organism and Milieu. Ed. by D. Rudnick, p. 253, New York: The Ronald Press.

Loomis, W. F. (1964). Microenvironmental control of sexual differentiation in hydra. J. exp. Zool. 156, 289.

Rafalko, J.S. (1947). Cytological observations on the amoeboflagellate Naegleria gruberi. J. Morphol. 81, 1.

Schuster, F. (1963). An electron microscope study of the amoeboflagellate Naegleria gruberi (Schardinger). II. The cyst stage. J. Protozool. 10, 313.

Scotr, W. W. (1939). Standard methods of chemical analysis. 5th ed. Ed. by N. H. Furman, vol. 2, p. 2072. New York: D. Van Nostrand Co.

Singe, B. N. (1941). The influence of different bacterial food supplies on the rate of reproduction in Colpoda steinii, and the factors influencing encystation. Ann. appl. Biol. 27, 65.

Thimann, K. V. (1963). The Life of Bacteria, 2nd ed, p. 265. New York: Macmillan Co.

Wirson, C. W. (1916). On the life-history of a soil amoeba. Univ. Calif. Publ. Zool. 16, 241. 